\title{
OLDER AND YOUNGER DRIVER PERFORMANCE AT COMPLEX INTERSECTIONS: IMPLICATIONS FOR USING PERCEPTION-RESPONSE TIME AND DRIVING SIMULATION
}

\author{
C.J. Edwards, J.I. Creaser, J.K. Caird, A.M. Lamsdale, \& S.L. Chisholm \\ Cognitive Ergonomics Research Laboratory \\ Department of Psychology \\ University of Calgary \\ Calgary, AB, Canada \\ E-mail: cjedward@ucalgary.ca
}

\begin{abstract}
Summary: Older drivers are at increased accident risk at intersections for a variety of maneuvers. To examine why, a study was conducted to assess older driver performance at complex intersections in a driving simulator. The University of Calgary Driving Simulator was used to test healthy older drivers (65-83, $M=71.4)$ and younger drivers (19-22, $M=20.7)$. Critical scenarios included the sudden appearance of a pedestrian in an intersection, a lastsecond yellow light, an unexpected change during a left, and a vehicle violating a stoplight. Older drivers had significantly higher perception response times (PRT) than younger drivers for the latter three of the four intersection scenarios. Analysis of specific maneuvers also revealed qualitative response differences between young and old groups. In contradiction to expectation, more older drivers ran the yellow light than younger drivers. The capability of older drivers to respond under time constraints is implicated. The utility of driving simulators to assess older driver performance at intersections was limited by the prevalence of simulator sickness.
\end{abstract}

\section{OLDER DRIVER INTERSECTION CRASHES AND PERFORMANCE}

Foremost among potential accident risks for older drivers are intersection collisions. At intersections, older drivers are over-represented in left turn and gap-acceptance crashes (see Caird \& Hancock, 2002 for review). Collision fatalities for a range of intersection maneuvers are more likely for those 75 years and older (Preusser et al., 1998). Frailty of older drivers, once in a crash, contributes to higher fatality rates (Hauer, 1988). A number of reasons for the increased accident involvement at intersections have been identified. For example, reductions in the useful field of view (Owsley et al., 1998) were found to be predictors of intersection crashes. Preusser et al. (1998) suggested that older drivers miss stop signs and stop lights at intersections.

Perception response time (PRT) by older drivers to traffic controls, other vehicles and pedestrians is another important driver performance measure at intersections. PRT is a measure of the length of time between the appearance of a hazard or threat and the initiation of braking. PRT is a fundamental component of many highway design equations, of accident reconstruction and it is used as a performance measure in driving research. Some researchers who have studied PRT have found age differences (Olson, 1996), whereas others have not (Lerner et al., 1995). Reconciling whether PRT can be used to describe age-related changes in intersection performance requires additional research. To gain a better understanding of the contributors to 
older driver intersection crashes, PRT was measured in a number of accident-likely intersections in a driving simulator. The advantage of using PRT to measure intersection performance is that results can be compared to a large body of laboratory and on-the-road research.

\section{METHOD}

\section{Participants}

Twelve older (65-83, $M=71.4)$ and twelve younger $(19-22, M=20.7)$ drivers were recruited. A valid driver's licence and an active driving record were required for participation. Participants were screened prior to entry into the study for visual and cognitive disease, certain medication uses, acuity, contrast sensitivity and proneness to simulator sickness. The younger drivers had an average of four years of driving experience, while the older drivers had an average of 52 years of experience. Older drivers reported average annual mileages of 20,916 kilometres per year in comparison to 15,791 kilometres per year for the younger group. The mean number of total accidents was higher for the older age group $(M=2.8, S D=3.4)$ than the younger group $(M=$ $0.83, S D=1.1$ ), which is a reflection of driving exposure. The number of accidents in the past five years is marginally lower for older drivers $(M=0.42, S D=0.9)$ compared to the young drivers $(M=0.75, S D=1.0)$. The numbers of driving violations for the older drivers were somewhat higher $(M=0.75, S D=1.2)$ compared to the younger drivers $(M=0.25, S D=0.62)$.

\section{Driving Simulator Hardware and Software}

The University of Calgary Driving Simulator (UCDS) is a 3-channel, 150-degree forward field of view vection simulator built by GlobalSim Corporation. A full description of the simulator and custom modifications is available in Caird, Edwards, Creaser and Horrey (2002). For this study, HyperDrive (v. 1.4.0) was used to develop the traffic environments and experimental scenarios for the driving simulator.

\section{Procedures}

Training and Experimental Scenarios. After visual testing, participants drove a training roadway section with three parts and an experimental section with four roadway sections. Instructions to turn or drive the posted speed limit were presented on the forward screen directly in front of the driver in white letters. The participant was also able to ask for clarification or help, if needed, through the simulator's communication system. Three training scenarios, which took 5 minutes to complete at $70 \mathrm{~km} / \mathrm{h}$, were used to familiarize participants with how the simulator operated. Four experimental routes, each with a critical event, were developed for this study. Each route consisted of a series of intersections where a critical event occurred at one intersection in the series. The locations of the critical events changed in each series of intersections to prevent participants from anticipating an event. Critical events included the sudden appearance of a pedestrian during a right turn (Pedestrian), a last-second yellow light (Yellow Light), the unexpected appearance of a pedestrian using a grey mask to induce change blindness (e.g., see O'Regan et al., 1999) (Dynamic Flicker), and a vehicle violating a red light while the participant had a green light (Vehicle Incursion). At each of the critical event intersections, other traffic, pedestrians and signs were present to increase the complexity of the visual field. 


\section{RESULTS}

Perception Response Time. Each participant's perception response time (PRT), in seconds, was determined for each event in the simulation study. PRT is defined as the interval of time between the appearance of some object or condition in the driver's field of view and the initiation of a response (Olson, 1996, p. 192). PRT was calculated based on any response made by the participants, whether the participants braked or accelerated. PRTs that could not be calculated due to lack of response or because the response was made in advance of an actual event, were not included in the analysis. As reflected in the degrees of freedom in the analysis below, 5 observations were lost altogether. Table 1 outlines the mean and standard deviations for PRT (in seconds), for both age groups and all events.

Table 1. PRT means and standard deviations (in seconds) for each event type and age group

\begin{tabular}{|l|c|c|}
\hline \multirow{2}{*}{\multicolumn{1}{|c|}{ Event type }} & \multicolumn{2}{|c|}{$\begin{array}{c}\text { Age Group } \\
\text { PRTs (s) (SD) }\end{array}$} \\
\cline { 2 - 3 } & 19 to 23 & 65 to 83 \\
\hline Pedestrian & $0.97(0.46)$ & $1.44(0.45)$ \\
\hline Yellow Light & $0.76(0.18)$ & $1.26(0.29)$ \\
\hline Dynamic Flicker & $0.62(0.41)$ & $1.40(0.58)$ \\
\hline Vehicle Incursion & $1.14(0.31)$ & $1.50(0.28)$ \\
\hline
\end{tabular}

A series of four independent sample t-tests were performed comparing event type PRT among each age group. Response to the pedestrian event was marginally significant between the young and old age groups, $M=0.97$ and $M=1.4$, respectively, $t(16)=-2.118, p=0.050$. For each scenario, the older age group had longer and more variable PRTs than the younger age group. PRT to the Yellow Light event was significantly different between the age groups, $t(17)=-$ $4.508, p=0.0001$, with the younger age group responding to the light change faster $(M=0.76)$ than the older age group $(M=1.26)$. The response to the Dynamic Flicker presentation and the sudden appearance of a pedestrian was statistically significant, $t(17)=-3.468, p=0.003$, again with the younger age group responding to the change faster $(M=0.62)$ than the older age group $(M=1.41)$. The slower response times of the older drivers may reflect uncertainty of how to respond to the appearance of the flickering gray mask. The vehicle incursion was the last event of the experimental trial and PRT was also significantly different between the two age groups, $t(18)=-2.715, p=0.014$. The group means are higher than other events for both age groups. The younger age group responded faster to the Vehicle Incursion event $(M=1.14)$ compared to the older age group $(M=1.50)$. A power analysis was performed to determine the sufficiency of the sample sizes (Lachin, 1981) and the Yellow Light, Dynamic Flicker and Vehicle Incursion results were reliable.

Driver responses to each event are described in Table 2. Comparison of specific maneuvers between young and old groups reveals qualitative response differences as well. 
Table 2. Response types to critical events by age group

\begin{tabular}{|c|l|c|c|c|}
\hline \multirow{2}{*}{$\begin{array}{c}\text { Age } \\
\text { group }\end{array}$} & \multicolumn{1}{|c|}{ Response type } & \multicolumn{3}{c|}{$\begin{array}{c}\text { Event Type } \\
(\%)\end{array}$} \\
\cline { 3 - 5 } & & $\begin{array}{c}\text { Yellow } \\
\text { Light }\end{array}$ & Dynamic Flicker & $\begin{array}{c}\text { Vehicle } \\
\text { Incursion }\end{array}$ \\
\hline \multirow{3}{*}{$\begin{array}{c}\text { Young } \\
\text { 19 to 22) }\end{array}$} & Braked & 50 & 58.3 & 83.3 \\
\cline { 2 - 5 } & Accelerated & 50 & 41.7 & - \\
\cline { 2 - 5 } & Braked but struck object & - & - & 8.3 \\
\cline { 2 - 5 } & Neither braked nor accelerated & - & - & 8.3 \\
\hline \multirow{3}{*}{$\begin{array}{c}\text { Older } \\
65 \text { to } 83)\end{array}$} & Braked & 25 & 58.3 & - \\
\cline { 2 - 5 } & Accelerated & 75 & 16.7 & 25 \\
\cline { 2 - 5 } & Braked but struck object & - & - & 33.3 \\
\cline { 2 - 5 } & Neither braked nor accelerated & - & 25 & \\
\hline
\end{tabular}

\section{DISCUSSION}

Perception Response Time and Response Patterns. Overall, PRTs were lower for the younger age group compared to the older age group. In the Yellow Light scenario, more younger drivers stopped for the light than older drivers. These results are contradictory to the expectation that younger drivers are more willing to run yellow lights. The decision by older drivers to continue through the intersection appears indicative of their inability to respond to the light quickly. The Vehicle Incursion scenario produced a differential mixture of responses across age groups. Most of the younger participants braked for the incurring vehicle, whereas older participants tended to make no response at all. One younger and two older drivers were struck by the red-light-running vehicle. The introduction of a gray mask for $80 \mathrm{~ms}$ in the Dynamic Flicker scenario was the first extension of the static flicker method (O'Regan et al., 1999) to a dynamic setting (Caird et al., 2002). The use of the dynamic flicker method to create effortful search may only be useful for determining search difficulties for static objects (e.g., signs and signals) than for objects in motion (e.g., pedestrians and vehicles). After the mask flicker stopped, once the pedestrian or vehicle moved, detection and responses followed subsequently. Familiarization during training would reduce the confusion produced by the flicker - especially for those in the older age group. The inclusion of the dynamic flicker method (DFM) into driving simulation has the potential to reveal specific scanning difficulties of drivers in complex scenarios. Although previous research has found mixed results on age differences in PRT in simulated and on-road driving, it is important to note that previous onroad and simulator studies that did not find age differences used very simple situations where other vehicles were not present (e.g., Lerner et al., 1995). This study examined complex intersections, with signs, pedestrians and other vehicles present. Therefore, the PRT results noted here may be more reflective of the attentional demands on older drivers than previous PRT studies. Furthermore, intersection design specifications for older drivers that use PRT often examine very simple situations, without taking into consideration the demands of a visually complex intersection on older drivers' attention and response times.

Simulator Sickness. Forty percent of recruited older participants did not complete the study due to symptoms of simulator sickness compared to $14 \%$ of younger participants. This dropout rate between younger and older adults was not significant when a chi-square test using Fisher's exact test for small sample sizes was conducted, $\chi^{2}(1)=2.62, p=.14$. The loss of our participants 
occurred despite screening for related symptoms such as a disposition for car or sea sickness (Kennedy et al., 1993). Anecdotal reports from researchers in Europe and the U.S. suggest that older drivers are more likely to be susceptible to simulator sickness. However, we were unable to find any quantitative or qualitative descriptions of older driver simulator sickness. Simulator or motion sickness are terms used to describe symptoms that participants feel and report after exposure to real or illusory motion (Stern \& Kock, 1996). Observable symptoms include sweating, pallor, repeated swallowing, increased head motion, and vomiting. Reported symptoms include disorientation, dizziness, and nausea. Where older drivers were required to drive straight and the forward field was limited to 50 degrees, 3 out of 24 or $12.5 \%$ of older participants chose to stop the simulation session (Caird et al., 2001), compared with 8 out of 20 in our simulator, which has 150 degree forward field. Both of these studies and other research (Stern \& Koch, 1996) suggest that women are more susceptible to simulator sickness than men. The loss of older participants due to simulator sickness may affect the representativeness of the driver PRT sample. In the worst case, those that drop out due to simulator sickness may be more at risk for intersection collisions.

\section{ACKNOWLEDGMENTS}

Ling Suen monitored and guided the project for the Transportation Development Centre (Transport Canada). Coben Christiansen and Tallia Richards also helped to run the participants in this study. Funding from the Canada Foundation for Innovation, Alberta Innovation and Science, the Centre for Transportation Engineering and Planning (C-TEP), and the University of Calgary provided the hardware, software, and infrastructure for the driving simulator.

\section{REFERENCES}

Caird, J.K., Edwards, C.J., Creaser, J.I., \& Horrey, W.J. (2002). Contributing factors to accidents at intersections for older drivers (Rep. No. TP13939E). Montreal, Canada: Transport Development Centre, Transport Canada.

Caird, J.K., \& Hancock, P.A. (2002). Left turn and gap acceptance accidents. In R.E. Dewar \& R. Olson (Eds.), Human factors in traffic safety (pp. 591-640). Tucson, AZ: Lawyers \& Judges Publishing.

Caird, J.K., Horrey, W.J., \& Edwards, C.J. (2001). Effects of conformal and non-conformal vision enhancement systems on older driver performance. Transportation Research Record, $1759,38-45$.

Hauer, E. (1988). The safety of older persons at intersections. In Special Report 218: Transportation in an Aging Society (Vol. 2, pp. 195-252), Washington, DC: Transportation Research Board, National Research Council.

Kennedy, R. S., Lane, N. E., Berbaum, K. S., \& Lilienthal, M. G. (1993). Simulator sickness questionnaire: An enhanced method for quantifying simulator sickness. International Journal of Aviation Psychology, 3(3), 203-220.

Lachin, J.M. (1981). Introduction to sample size determination and power analysis for clinical trials. Controlled Clinical Trials, 2, 93-113.

Lerner, N.D., Huey, R.W., McGee, H.W., \& Sullivan, A. (1995). Older driver perceptionreaction time for intersection sight distance and object detection (Final Rep., Vol. I, No. RD93-168). McLean, VA: Federal Highway Administration. 
Olson, P.L. (1996). Forensic aspects of driver perception and response. Tuscon, AZ: Lawyers \& Judges Publishing.

O’Regan, J.K., Rensink, R.A., \& Clark, J.J. (1999). Change blindness as a result of "mudsplashes". Nature, 398, 34.

Owsley, C., Ball, K., McGwin, G., Jr., Sloane, M.E., Roenker, D.L., White, M.F., \& Overley, E.T. (1998). Visual processing impairment and risk of motor vehicle crash among older adults. Journal of the American Medical Association, 279(14), 1083-1088.

Preusser, D.F., Williams, A.F., Ferguson, S.A., Ulmer, R.G., \& Weinstein, H.B. (1998). Fatal crash risk of older drivers at intersections. Accident Analysis and Prevention, 30(2), 151-159.

Stern, R.M., \& Koch, K.L. (1996). Motion sickness and differential susceptibility. Current Directions in Psychological Research, 5(4), 115-120. 